\title{
Reconstruction Metrics in Atom Probe Tomography
}

\author{
BP Geiser ${ }^{1}$, DA Reinhard ${ }^{1 *}$, JH Bunton ${ }^{1}$, TR Payne ${ }^{1}$, KP Rice $^{1}$, Y Chen $^{1}$ and DJ Larson ${ }^{1}$ \\ 1. CAMECA Instruments Inc., Madison, WI, USA. \\ * Corresponding author: david.reinhard@ametek.com
}

The standard reconstruction algorithm [1,2] for atom probe tomography relies on evolving a simple spherical model with acquisition time. Initial geometry and projection parameters are assumed and updated with estimated acquired volume. The geometry is typically driven either by use of the specimen voltage history, as a proxy for the apex size, or by the assumption of a fixed specimen shank angle. In most cases the evolution proceeds without any feedback to correct these assumptions, so errors in the initial geometry or projection properties propagate and tend to build. Errors like this can result in reconstructions that are distorted by scaling factors.

While developing a history of absolute metric values for specific systems should help in understanding the relative quality of a specific reconstruction, comparing actual data metrics to metrics obtained from simulated (either realistic field evaporation/projection or ideal evaporation/projection) data may provide even more information about the kinds of errors present in data before it is further analyzed.

Crystallographic systems present many opportunities to assess reconstruction quality. Fourier transforms have been used to calibrate reconstruction parameters [3]. Spatial distribution maps can be used to estimate plane spacing [4]. Several novel algorithms [5, 6] have been proposed to measure the variation of planar spacing with depth, feeding that back into the reconstruction, or explicitly using postreconstruction measurements of that variation to qualify the reconstruction.

In structured materials, it is common to experience more local distortions in the reconstruction due to more extreme apex reshaping reflecting differences in evaporation fields of different materials. In these cases, different metrics may be more useful. For example, distributions of local relative density errors [7] can provide quantitative means to assess the effectiveness of different reconstruction parameter-sets or techniques. Here for each center ion we measure the ratio of the ranged volume of the nearest $\mathrm{N}$ ions to the volume of the sphere centered on the ion containing the $\mathrm{N}^{\text {th }}$ nearest neighbor ion. Figure 1 shows a reconstruction and localized relative densities. Figure 2 shows a mass spectrum histogram and the distribution of relative density values computed with this metric.

$$
\rho_{m}=\frac{\sum_{N} V_{\text {ranged }}^{i}{ }_{\varepsilon}}{\frac{4}{3} \pi r_{N}^{3}}
$$

Access to localized data quality indicators can inform an analyst's decision both on the appropriateness of a given reconstruction algorithm, and also on potential changes to the acquisition process to optimize future experiments. For example, drastic variations in charge-state ratio throughout an acquisition can indicate a deviation from a spherical tip apex and the need for a revised laser pulse energy protocol. With the release of CAMECA's new APT file format [8], which provides the flexibility to allow additional fields per ion, the visualization of quality metrics will become practical allowing increased diagnostic data for APT reconstructions. 


\section{References:}

[1] D Blavette et al., Revue Phys. Appl. 17 (1982), p. 435.

[2] P Bas et al., Applied Surface Science 87/88 (1995), p. 298.

[3] B Gault et al., Journal of Applied Physics 105 (2009), p. 034913.

[4] E Marquis et al., Microscopy and Microanalysis 13 (2007), p. 196.

[5] C Hatzoglou et al., Ultramicroscopy 197 (2018), p. 72.

[6] A Day et al., Microscopy and Microanalysis, In Press.

[7] L Stephenson et al., Microscopy and Microanalysis 13 (2007), p. 448.

[8] D Reinhard et al., Improved Data Analysis with IVAS 4 and AP Suite (This Proceeding).
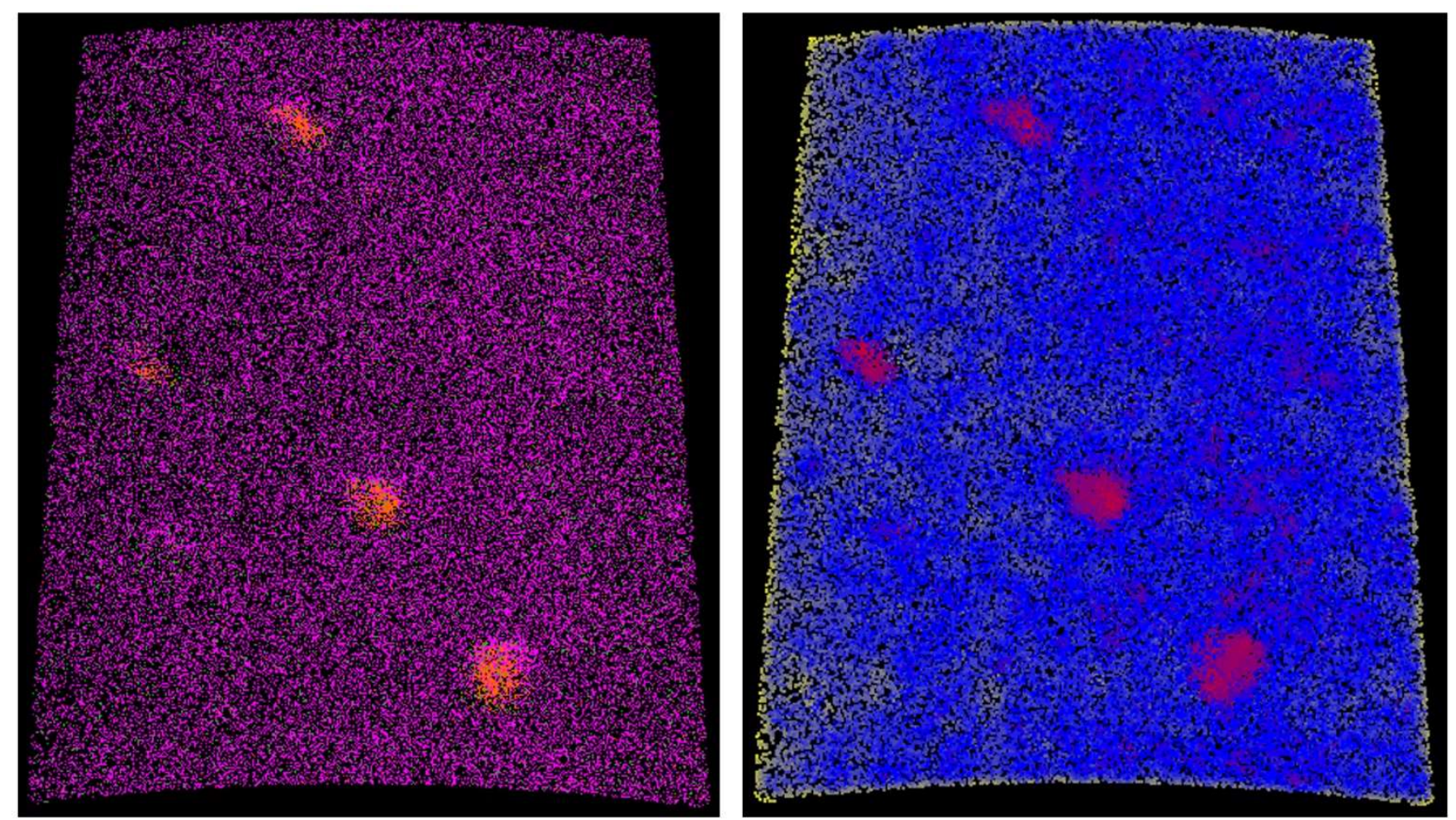

Figure 1. (left) APT analysis of copper particles in an iron matrix. (right) Visualization of the density metric with color values as indicated in Fig. 2.
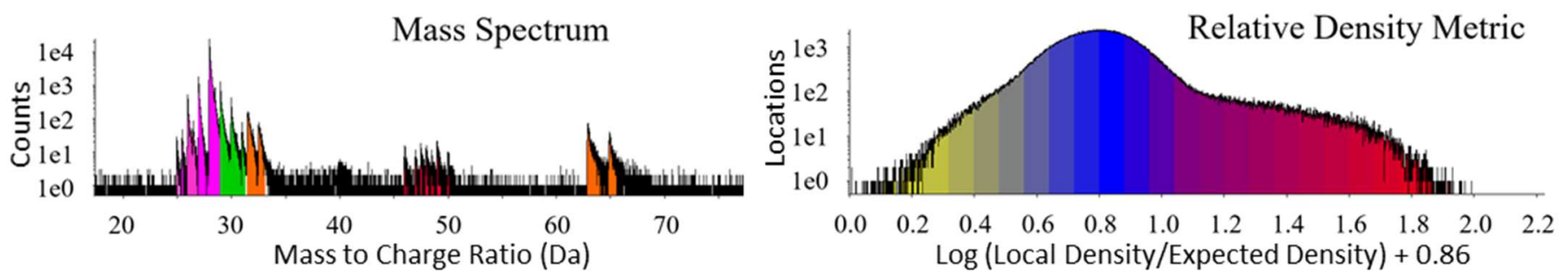

Figure 2. Mass spectrum of the copper in iron specimen in Fig. 1. Distribution of the logarithm of the density metric. Since the metric is a ratio, this helps equally represent very low metric values. The distribution has been shifted positively by 0.86 for visualization. 